\title{
MAPPINGS BY MEANS OF SYSTEMS OF ANALYTIC FUNCTIONS OF SEVERAL COMPLEX VARIABLES
}

\author{
W. T. MARTIN
}

1. Introduction. An analytic mapping of a domain $D$ in the space $E_{2 k}$ of $k$ complex variables $z_{1}, \cdots, z_{k}$ is a mapping defined by

$$
T: z_{j}^{\prime}=f_{j}\left(z_{1}, \cdots, z_{k}\right), \quad j=1, \cdots, k,
$$

where the $f_{j}(z)$ are analytic in $D$. We shall consider only univalent (schlicht) domains $D$ contained in the finite portion of the space $E_{2 k}$. An important result in the theory of analytic mappings states that the mapping is topological (that is, $1-1$ and bi-continuous) if, and only if, the Jacobian

$$
J(z) \equiv \partial\left(f_{1}, \cdots, f_{k}\right) / \partial\left(z_{1}, \cdots, z_{k}\right)
$$

is different from zero at each point of $D$ (Carathéodory [12]). ${ }^{1}$

An analytic mapping $T$ is called an inner mapping of a domain $D$ if $T D \subset D$. It is called an automorphism of $D$ if $T$ is $1-1$ and if $T D=D$.

In 1907 Poincaré [17] showed that, given two domains $D$ and $D^{\prime}$, it is not always possible to map $D$ onto $D^{\prime}$ analytically. Since that time, several general problems have been considered. One of these problems is to indicate some general rules which tell whether or not two given domains can be mapped analytically upon each other. A second problem is to determine a family of special domains, in terms of some simple properties, the family to be such that every domain can be mapped analytically onto one of these special domains.

In this talk I shall deal with a special case of the first of these two problems together with certain results on the second problem. The work on the first problem which will be presented is based upon Henri Cartan's theory of mappings of domains onto domains of circular type. The work given on the second problem is based upon Bergman's theory of representative domains. In preparing this talk, I have used freely the excellent résumé on analytic mapping contained in the book on several complex variables by Behnke and Thullen [4]. I have also used freely material from the manuscript by Bochner and the speaker of a book on several complex variables, now in preparation [10]. I am indebted to Professor Bochner for permission to use

An address delivered before the New York meeting of the Society on October 30, 1943, by invitation of the Program Committee; received by the editors November 9, 1943.

1 Numbers in brackets refer to the Bibliography at the end of the paper. 
material from this manuscript. The approach used in much of this talk follows the approach of that manuscript, and in addition certain so far unpublished results from it are given here.

Let $D$ be a domain containing the origin, and let (1.1) be an analytic mapping of $D$ onto a domain $D^{\prime}$, with a fixed origin. We shall frequently write (1.1) in the form

$$
\begin{aligned}
& T: z_{j}^{\prime}=a_{1 j} z_{1}+\cdots+a_{k j} z_{k}+\text { (higher powers), } \\
& \qquad j=1, \cdots, k,
\end{aligned}
$$

to mean that the analytic functions $f_{j}(z)$ defining $T$ have the expansions

$$
f_{j}(z)=a_{1 j} z_{1}+\cdots+a_{k j} z_{k}+\text { (higher powers) }
$$

in a neighborhood of the origin.

Again, if $D$ is a domain containing the origin and if $f\left(z_{1}, \cdots, z_{k}\right)$ is analytic in $D$ we shall sometimes have occasion to develop $f$ in the neighborhood of the origin in terms of homogeneous polynomials (a diagonal series)

$$
f\left(z_{1}, \cdots, z_{k}\right)=\sum_{n=0}^{\infty} P_{n}\left(z_{1}, \cdots, z_{k}\right) .
$$

The expression $P_{n}(z)$ is a homogeneous polynomial of degree $n$ and is given by

$$
P_{n}(z)=\frac{1}{2 \pi} \int_{0}^{2 \pi} f\left(z_{1} e^{i \theta}, \cdots, z_{k} e^{i \theta}\right) e^{-i n \theta} d \theta
$$

for $z$ in a sufficiently small neighborhood $N$ of the origin. From (1.6) we see that if $|f(z)| \leqq M$ in $N$, then

$$
\left|P_{n}(z)\right| \leqq M \text { for } z \in N, \quad n=0,1,2, \cdots \text {. }
$$

2. Some uniqueness properties of analytic mappings. H. Cartan [13] and C. Carathéodory [12] have proved two very elegant results on the uniqueness of analytic mappings. We shall give these two results here:

TheOREM 1 (CARTAN). Let $D$ be a domain containing the origin and let $T$ be an analytic mapping of $D$ into a bounded domain $D^{\prime} \subset D$. If the linear part of $T$ is the identity, that is, if $T$ has the form

(2.1) $T: z_{j}^{\prime}=f_{j}\left(z_{1}, \cdots, z_{k}\right)=z_{j}+$ (higher powers), $j=1, \cdots, k$, then $T$ is actually the identity 


$$
f_{j}(z) \equiv z_{j}, \quad j=1, \cdots, k .
$$

Proof. Expand the $f_{j}(z)$ in terms of homogeneous polynomials:

$$
f_{j}(z)=z_{j}+P_{r_{j}}^{j}(z)+P_{r_{j}+1}^{j}(z)+\cdots
$$

with $r_{j}$ an integer not less than 2. In writing $f_{j}(z)$ in this form, we are assuming that the polynomials $P_{n}^{j}$ of degree $n$ for $1<n<r_{j}$ all vanish identically. Now iterate the mapping. We find that $T^{2}$ has the expansion

$$
T^{2}: z_{j}^{\prime \prime}=z_{j}^{\prime}+P_{r j}^{j}\left(z^{\prime}\right)+\cdots=z_{j}+2 P_{r_{j}}^{j}(z)+\cdots,
$$

and in general

$$
T^{m}: z_{j}^{(m)}=z_{j}+m P_{r_{j}}^{j}(z)+\cdots .
$$

Now $T$ maps $D$ into a bounded domain $D^{\prime} \subset D$, hence the same is true of all the iterates $T^{2}, T^{3}, \ldots$. Thus the mapping functions

$$
f_{j}^{(m)}(z)=z_{j}+m P_{r_{j}}^{j}(z)+\cdots
$$

are uniformly bounded in $D$. By the result given at the end of $\S 1$, this implies that

$$
\left|m P_{r_{j}}^{j}(z)\right| \leqq M, \quad \text { for } j=1, \cdots, k ; m=1,2, \cdots,
$$

for $z$ in a sufficiently small neighborhood of the origin. This implies that $P_{r_{j}}^{f}(z) \equiv 0$ and hence (2.2) holds.

Carathéodory's uniqueness theorem follows almost immediately from Cartan's theorem.

THEOREM 2 (CARATHÉODORY). There exists at most one 1-1 analytic mapping of a domain $D$ onto a bounded domain $D^{\prime}$ which maps a point $O$ of $D$ onto a point $O^{\prime}$ of $D^{\prime}$ and whose first partial derivatives in $O$ have prescribed values.

Proof. If $S$ and $T$ are two such mappings, then the mapping $S T^{-1}$ is a mapping of $D^{\prime}$ onto itself and the hypotheses of Theorem 1 are satisfied. Thus

$$
S T^{-1}=I \text { or } S=T .
$$

An important corollary has been derived from Theorem 2. First we make a definition. A circular domain is a domain which is mapped onto itself by all the mappings

$$
T(\theta): \quad z_{j}^{\prime}=e^{i \theta} z_{j}, \quad j=1, \cdots, k ; 0 \leqq \theta<2 \pi .
$$

A circular domain is called proper if it contains the origin. Circular 
domains were introduced by Behnke [1] and Carathéodory [11] and have been investigated by various writers. We shall return to them in $\$ 4$. For the present we give only the following result.

COROLLARY. Every automorphism of a proper bounded circular domain which leaves the origin fxed is a homogeneous linear transformation.

Proof. Let

$$
A: \quad z_{j}^{\prime}=\sum_{n=1}^{\infty} P_{n}^{j}\left(z_{1}, \cdots, z_{k}\right), \quad j=1, \cdots, k,
$$

be any such automorphism. Then $T(\theta) A$ has the form

$$
T(\theta) A: \quad z_{j}^{\prime}=\sum_{n=1}^{\infty} e^{i \theta} P_{n}^{j}\left(z_{1}, \cdots, z_{k}\right)
$$

and $A T(\theta)$ has the form

$$
A T(\theta): \quad z_{j}^{\prime}=\sum_{n=1}^{\infty} P_{n}^{j}\left(z_{1} e^{i \theta}, \cdots, z_{k} e^{i \theta}\right)=\sum_{n=1}^{\infty} e^{i n \theta} P_{n}^{i}(z) .
$$

Since $D$ is circular and $A$ is an automorphism, both transformations $T(\theta) A$ and $A T(\theta)$ are automorphisms of $D$, with the origin fixed. Also, both have the same linear parts. Hence, by Theorem 2, they are the same. This can happen, however, only if $P_{n}^{\prime}(z) \equiv 0$ for $n>1$, that is only if $A$ is linear.

The corollary has been proved by Behnke [2], Bergman [5], H. Cartan [13], and Welke [18]. Bergman's proof, which is by a different method, will be given in $\$ 5$.

3. Inner mappings and automorphisms with a fixed point. Cartan's theorem tells us that if we have an inner analytic mapping $T$ of a domain $D$ containing the origin into a bounded domain $D^{\prime}$, and if the linear part of $T$ is the identity, then $T$ is actually the identity. Now suppose we have a general inner analytic mapping of a bounded domain $D$ with the origin as a fixed point:

$$
T: \quad z_{j}^{\prime}=a_{1 j} z_{1}+\cdots+a_{k j} z_{k}+\cdots, \quad j=1, \cdots, k .
$$

Denoting by $\Delta_{T}$ the value of the functional determinant at the origin:

$$
\Delta_{T}=\left|a_{i j}\right|_{i, j=1} \ldots, k,
$$

Cartan [14] and Carathéodory [12] have shown that 


$$
\left|\Delta_{T}\right| \leqq 1
$$

holds for every inner mapping, with the equality

$$
\left|\Delta_{T}\right|=1
$$

holding if and only if $T$ is an automorphism. This result is very important in the theory of analytic mapping and will be useful to us in a later section.

The result has recently been generalized a little by Bochner and the speaker [10]. Denoting by $\lambda_{1}, \cdots, \lambda_{k}$ the characteristic roots of the matrix $\left(a_{i j}\right)$, they have shown that

$$
\left|\lambda_{1}\right| \leqq 1, \cdots,\left|\lambda_{k}\right| \leqq 1
$$

holds for every inner transformation (3.1) and

$$
\left|\lambda_{1}\right|=\cdots=\left|\lambda_{k}\right|=1
$$

holds if and only if $T$ is an automorphism.

We shall not give the proofs of these results.

4. Groups of automorphisms of a bounded domain with a fixed point. Near the end of $\$ 2$ we introduced the notion of a circular domain, a domain which with a point $\left(z^{0}\right)$ contains also all the points

$$
\left(z_{1}^{0} e^{i \theta}, \cdots, z_{k}^{0} e^{i \theta}\right), \quad 0 \leqq \theta<2 \pi .
$$

Cartan [13] has generalized this notion as follows: Let $\left(m_{1}, \cdots, m_{k}\right)$ be $k$ integers (positive, negative, or zero). Then an $\left(m_{1}, \cdots, m_{k}\right)$ circular domain is one which, with a point $\left(z^{0}\right)$, contains all the points

$$
\left(z_{1}^{0} e^{i m_{1} \theta}, \cdots, z_{k}^{0 i m_{k} \theta}\right), \quad 0 \leqq \theta<2 \pi .
$$

The domain is called proper if it contains the origin.

The domains of circular type play in several respects the role of the circle in one complex variable. It is, therefore, natural to question whether every bounded domain $D$ can be mapped analytically onto some domain of circular type, or if not, which ones can. In the first place, an $\left(m_{1}, \cdots, m_{k}\right)$-circular domain admits an infinite number of automorphisms with the origin as a fixed point, namely those of (4.2). Therefore, if it is possible to map analytically a given domain $D$ onto an $\left(m_{1}, \cdots, m_{k}\right)$-circular domain, the domain $D$ must admit an infinite number of automorphisms leaving an interior point fixed. Cartan [13] (for $k=2$ ) has shown that this condition is also sufficient. Following the résumé given by Behnke and Thullen [4], we shall give a brief outline of Cartan's argument. 
THEOREM 3 (CARTAN). The group $G$ of all automorphisms of a bounded domain $D$ which leave the origin fixed $(O \in D)$ is isomorphic with a compact group $\Gamma$ of homogeneous linear transformations; the determinant of each transformation of $\Gamma$ is of absolute value unity.

By Carathéodory's theorem, Theorem 2, the group $\Gamma$ consists merely of the linear parts of the members of $G$, and by (3.4) the determinant of each transformation of $G$ is of absolute value unity.

An infinite compact group $\Gamma$ of homogeneous linear transformations (in the space of two complex variables) always leaves invariant an Hermitian form

$$
A w \bar{w}+B z \bar{z}+C w \bar{z}+\bar{C} \bar{w} z
$$

(Weyl [19]). By a suitable linear transformation $L$ this Hermitian form will be transformed into a normalized form

$$
w \bar{w}+z \bar{z}
$$

which is invariant under the corresponding group $\Gamma^{*}=L \Gamma L^{-1}$.

ThEOREM 4 (Cartan). Every infinite compact group of homogeneous linear transformations, which leave invariant the form (4.4), contains a subgroup of the form

$$
T_{m, p}(\theta): \quad w^{\prime}=w e^{i m \theta}, \quad z^{\prime}=z e^{i p \theta}, \quad 0 \leqq \theta<2 \pi .
$$

Let $G_{0}$ be the subgroup of $G$ which is isomorphic to the group $\left\{T_{m, p}(\theta)\right\}$. Denoting by $A(\theta)$ the isomorph in $G$ of $T_{m, p}(\theta)$, we know that $A(\theta)$ must have the form

$$
A(\theta): \begin{aligned}
w^{\prime} & =f(w, z ; \theta)=e^{i m \theta} w+\cdots, \\
z^{\prime} & =g(w, z ; \theta)=e^{i p \theta_{z}}+\cdots .
\end{aligned}
$$

Now define

$$
\begin{aligned}
& F(w, z)=\frac{1}{2 \pi} \int_{0}^{2 \pi} f(w, z ; \theta) e^{-i m \theta} d \theta, \\
& G(w, z)=\frac{1}{2 \pi} \int_{0}^{2 \pi} g(w, z ; \theta) e^{-i p \theta} d \theta .
\end{aligned}
$$

Then Cartan showed that except possibly for certain exceptional cases the mapping

$$
S: \quad w^{\prime}=F(w, z), \quad z^{\prime}=G(w, z)
$$

is a 1-1 analytic mapping of $D$ onto a proper $(m, p)$-circular domain 
with $S O=O$. (It is easily seen that the linear part of $S$ is the identity.) In the exceptional cases, Cartan showed that $S$ can be replaced by a mapping $\tilde{S}$ which has the requisite properties. Hence, we have:

TheOREM 5 (CARTAN). If a bounded domain $D$ admits an infinite group $G$ of automorphisms with the origin as a fixed point $(O \in D)$, then $D$ can be mapped in a 1-1 analytic manner on a bounded proper $(m, p)$ circular domain $D^{\prime}$ with the origin going into the origin.

The structure of the group tells one what type of circular domain it will go into.

It is known that there exist bounded domains $D$ which possess the following property: given any point $P$ in $D$, the automorphisms of $D$ which leave $P$ fixed are finite in number [13]. Hence, not every bounded domain can be mapped onto an $(m, p)$-circular domain.

This concludes our résumé of Cartan's work. Certain aspects of it have been generalized. We shall indicate this generalization.

Let $D$ be a bounded domain in $E_{2 k}$ containing the origin, and let $G$ be a group of automorphisms of $D$ with the origin as a fixed point. If $T(\alpha), T(\beta)$, and so on, are elements of $G$, and if we write the expansion of $T(\alpha)$ in the form

$$
\begin{aligned}
T(\alpha): z_{j}^{\prime}= & a_{1}^{j}(\alpha) z_{1}+\cdots+a_{k}^{j}(\alpha) z_{k} \\
& \quad+\sum_{n_{1}+\cdots+n_{k} \geqq 2} a_{n_{1}}^{j} \cdots n_{k}(\alpha) z_{1}^{n_{1}} \cdots z_{k}^{n_{k}},
\end{aligned}
$$

then Carathéodory's uniqueness theorem, Theorem 2, states that if

$$
a_{i}^{i}(\alpha)=a_{i}^{i}(\beta), \quad i, j=1, \cdots, k,
$$

then

(4.11) $\quad a_{n_{1} \cdots n_{k}}^{j}(\alpha)=a_{n_{1} \cdots n_{k}}^{j}(\beta), \quad j=1, \cdots, k ; n_{1}+\cdots+n_{k} \geqq 2$.

Actually a little more than this has recently been proved [10], namely:

Theorem 6 (Bochner ANd MARTin). If $T(\alpha), T(\beta)$, and so on, are elements of a group $G$ of automorphisms of a bounded domain $D$ with the origin as a fixed point, and if $\left(j, n_{1}, \cdots, n_{k}\right)$ is any fixed multiindex, then corresponding to any $\epsilon>0$, there exists $a \delta>0$ such that

$$
\sum_{\rho, \sigma=1}^{k}\left|a_{\sigma}^{\rho}(\alpha)-a_{\sigma}^{j}(\beta)\right| \leqq \delta
$$

implies

$$
\left|a_{n_{1} \cdots n_{k}}^{j}(\alpha)-a_{n_{1} \cdots n_{k}}^{i}(\beta)\right| \leqq \epsilon .
$$


(The a's are the coefficients in the expansion of the T's as in (4.9).)

The proof of this theorem uses strongly the uniqueness theorem of Carathéodory. We shall omit the proof. By use of Theorem 6, Bochner and the speaker [10] have obtained the following theorem which is related to Cartan's theorem, Theorem 5.

THEOREM 7. If $\{T(\alpha)\}$ is a group $G$ of automorphisms of a bounded domain $D$ with the origin as a fxed point, then there exists a change of coordinates $S$ in the neighborhood of the origin

$$
S: z_{j}^{\prime}=z_{j}+(\text { higher powers) }, \quad j=1, \cdots, k,
$$

such that the given automorphisms are linear transformations in the new coordinates,

$$
L(T(\alpha))=S T(\alpha) S^{-1} .
$$

We shall outline the proof of this theorem as given in [10].

By Theorem 2 each $T(\alpha)$ is uniquely determined by $L(T(\alpha))$ and hence by the $k^{2}$ complex numbers $a_{\mu}^{j}(\alpha), \mu, j=1, \cdots, k$. Thus, every element of $G$ can be identified with a point in Euclidean space $E$ of $2 k^{2}$ dimensions, and $G$ itself with a bounded point set in $E$. Also since

$$
L(T(\alpha) \cdot T(\beta))=L(T(\alpha)) \cdot L(T(\beta)),
$$

the group product $\beta \alpha$ of any two elements $\alpha, \beta$ of $G$ is given by

$$
a_{m}^{i}(\beta \alpha)=\sum_{\mu=1}^{k} a_{\mu}^{i}(\beta) a_{m}^{\mu}(\alpha), \quad j, m=1, \cdots, k .
$$

Thus in the ordinary Euclidean topology of $E$, the group product $\beta \alpha$ is a continuous function of $\beta$ and $\alpha$.

Now, since $G$ is bounded, there exists an additive measure $\mu$ on $G$ with the following properties among others:

(i) the measure of the total set $G$ is 1 ,

(ii) the measure is group invariant, and

(iii) every function $f(\alpha)$ on $G$ which is uniformly continuous on $G$ (in the topology of $E$ ) is integrable.

Now Theorem 6 states that each coefficient of $T(\alpha)$ is uniformly continuous on $G$. Hence, by property (iii) each coefficient of $T(\alpha)$ is in tegrable relative to our measure. It follows that the transformation $L\left(T\left(\alpha^{-1}\right)\right) \cdot T(\alpha)$ is measurable and it can be shown that the desired change of coordinates $S$ is given by

$$
S=\int_{G} L\left(T\left(\alpha^{-1}\right) \cdot T(\alpha) d \mu(\alpha) .\right.
$$


5. Bergman's representative domains. In connection with the second problem of the theory of analytic mapping mentioned in the introduction, Bergman [5] has introduced the notion of a representative domain of a class of equivalent domains, and in a series of papers he has developed several interesting aspects of the theory. A portion of his work has been simplified by Welke [18] and parts of it have been summarized by Behnke and Thullen [4] and by Bochner and the speaker [10]. In this section we shall follow the latter summary for the most part.

First we give two definitions due to Bergman.

Normalized mapping-An analytic mapping of a domain $D$ containing the origin is called normalized (with respect to the origin) if its linear part is the identity.

Equivalent domains-Two domains $D$ and $D^{\prime}$ are called equivalent if they can be mapped onto each other by a 1-1 analytic normalized mapping.

We shall use these two notions later in this section.

Let $D$ be a domain in $E_{2 k}$ and denote by $\mathcal{L}_{2}$ the set of all functions $f\left(z_{1}, \cdots, z_{k}\right)$ analytic in $D$ and such that the norm

$$
\|f\|=\left[\int_{D}|f(z)|^{2} d \omega_{z}\right]^{1 / 2}
$$

is finite $\left(d \omega_{z}=d x_{1} d y_{1} \cdots d x_{k} d y_{k}\right)$.

If $D$ has finite volume then every bounded analytic function in $D$ belongs to $\mathcal{L}_{2}$. If $D$ is also bounded this includes all polynomials. For the sake of simplicity, we restrict ourselves to bounded domains $D$. The space $\mathcal{L}_{2}$ is then a Hilbert space.

Now take a point in $D$, call it the origin and expand all functions $f(z)$ of $\mathcal{L}_{2}$ in power series about the origin. It can be shown that there exists a unique minimal function

$$
f_{0}(z)=1+\text { (higher powers) }
$$

such that

$$
\left\|f_{0}\right\|<\|f\|
$$

for every $f$ of $\mathcal{L}_{2}$ with the development

$$
f(z)=1+\text { (higher powers). }
$$

The same is true for functions of the form

$$
f_{j}(z)=z_{j}+\text { (higher powers), }
$$


that is for each $j=1, \cdots, k$ there exists a unique function $f_{j}(z)$ of $\mathcal{L}_{2}$ for which

$$
\left\|f_{j}\right\|<\|f\|
$$

holds for every $f \in \mathcal{L}_{2}$ in $D$ with the development

$$
f(z)=z_{j}+\text { (higher powers). }
$$

Bergman has shown that these $(k+1)$-minimal functions possess some beautiful forms of invariance. Consider a 1-1 normalized analytic transformation

$$
z_{j}=z_{j}\left(z_{1}^{\prime}, \cdots, z_{k}^{\prime}\right)=z_{j}^{\prime}+\text { (higher powers) }
$$

from $D$ onto a domain $D^{\prime}$ with nonvanishing Jacobian

$$
J\left(z^{\prime}\right)=\partial\left(z_{1}, \cdots, z_{k}\right) / \partial\left(z_{1}^{\prime}, \cdots, z_{k}^{\prime}\right) .
$$

Now, by the ordinary transformation of variables

$$
\int_{D}|f(z)|^{2} d \omega_{z}=\int_{D^{\prime}}\left|f\left(z\left(z^{\prime}\right)\right)\right|^{2}\left|J\left(z^{\prime}\right)\right|^{2} d \omega_{z^{\prime}}
$$

and it is not hard to see that

$$
f(z) \leftrightarrow f\left(z\left(z^{\prime}\right)\right) J\left(z^{\prime}\right)
$$

is a 1-1 transformation of $\mathcal{L}_{2}(z)$ onto $\mathcal{L}_{2}\left(z^{\prime}\right)$. Also since

$$
J\left(z^{\prime}\right)=1+\text { (higher powers) }
$$

we have

$$
f_{j}^{D}\left(z\left(z^{\prime}\right)\right) \equiv f_{j}^{D^{\prime}}\left(z^{\prime}\right) J\left(z^{\prime}\right), \quad j=0,1, \cdots, k,
$$

where $f_{j}^{D}(z)$ and $f_{j}^{D^{\prime}}\left(z^{\prime}\right)$ are the minimal functions for $D$ and $D^{\prime}$ respectively. Hence at least in a neighborhood of the origin

$$
f_{i}^{D}\left(z\left(z^{\prime}\right)\right) / f_{0}^{D}\left(z\left(z^{\prime}\right)\right) \equiv f_{j}^{D^{\prime}}\left(z^{\prime}\right) / f_{0}^{D^{\prime}}\left(z^{\prime}\right), \quad j=1, \cdots, k,
$$

and thus these quotients are absolute invariants. Omitting the superscript $D$, we denote these functions by $w_{1}(z), \cdots, w_{k}(z)$ and we observe that they have the expansion

$$
w_{j}(z)=z_{j}+\text { (higher powers), } \quad j=1, \cdots, k .
$$

Now assume that our domain $D$ is such that for $D$ these $k$ functions have the special form

$$
w_{j}(z) \equiv z_{j}, \quad j=1, \cdots, k
$$


Transcribe them by (5.8) onto $D^{\prime}$. Being invariants they go over into

$$
w_{j}^{\prime}\left(z^{\prime}\right)=z_{j}\left(z_{1}^{\prime}, \cdots, z_{k}^{\prime}\right)=z_{j}^{\prime}+\text { (higher powers). }
$$

Hence they cannot have the specialized form (5.16) in the domain $D^{\prime}$ unless the transformation (5.8) is the identity $z_{j} \equiv z_{j}^{\prime}$. In other words the specialized form (5.16) cannot occur for more than one domain $D$ in a class of equivalent domains and thus the specialized form (5.16) singles out the domain $D$ from all other domains $D^{\prime}$ which are equivalent to $D$ under a 1-1 analytic mapping which preserves the origin. This specialized domain is called the representative of its class by Bergman.

Now let $K$ be a class of equivalent domains and assume that the class has a representative domain $R$. Then each domain $D$ of $K$ can be mapped onto $R$ in a 1-1 manner by a normalized mapping. Denote this mapping by

$$
T: z_{j}^{\prime}=g_{j}(z), \quad z \in D,
$$

and consider the mapping

$$
S: \quad z_{j}^{\prime}=w_{j}^{D}(z)
$$

where the $w_{j}^{D}(z)$ are the invariant quotients for $D$. We do not assume that $S$ is $1-1$. But by the invariance we know that

$$
w_{j}^{D}\left[g_{1}^{-1}\left(z^{\prime}\right), \cdots, g_{k}^{-1}\left(z^{\prime}\right)\right] \equiv w_{j}^{R}\left(z^{\prime}\right),
$$

and since $R$ is representative

$$
w_{j}^{R}\left(z^{\prime}\right) \equiv z_{j}^{\prime}, \quad j=1, \cdots, k .
$$

Hence

$$
w_{j}^{D}\left[g_{1}^{-1}\left(z^{\prime}\right), \cdots, g_{k}^{-1}\left(z^{\prime}\right)\right] \equiv z_{j}^{\prime}
$$

that is

$$
\underset{w_{j}}{D}(z) \equiv g_{j}(z)
$$

Thus $S$ gives a 1-1 normalized mapping of $D$ onto $R$.

This yields a very beautiful result due to Bergman.

THEOREM 8 (BERGMAN). Let $K$ be a class of equivalent domains, equivalent with respect to 1-1 normalized analytic mappings. For $D \in K$ let

$$
f_{0}^{D}(z)=1+\text { (higher powers) }
$$




$$
f_{j}^{D}(z)=z_{j}+(\text { higher powers }), \quad j=1, \cdots, k,
$$

be the $k+1$ (unique) minimal functions for $D$ and define

$$
w_{i}^{D}(z)=f_{i}^{D}(z) / f_{0}^{D}(z), \quad j=1, \cdots, k .
$$

There exists at most one domain $R$ of $K$ for which the functions $w_{j}^{R}(z)$ have the specialized form

$$
w_{j}^{R}(z) \equiv z_{j}, \quad j=1, \cdots, k .
$$

If there is actually such a domain $R$ then every domain $D$ of $K$ can be mapped onto $R$ by a 1-1 normalized analytic mapping, and the mapping which does this is given by

$$
z_{j}^{\prime}=w_{j}^{D}(z), \quad j=1, \cdots, k ; z \in D .
$$

It can easily be seen that there is a representative domain if for at least one domain $D$ of $K$ the mapping

is $1-1$.

$$
z_{j}^{\prime}=w_{j}^{D}(z)
$$

Bergman has observed that every bounded proper circular domain $\Delta$ is a representative domain. To see this write the minimal function $f_{0}(z)$ for a bounded proper circular domain $\Delta$ in the form

$$
f_{0}(z)=1+P_{1}(z)+P_{2}(z)+\cdots,
$$

where $P_{n}(z)$ is a homogeneous polynominal of degree $n$. Then

$$
f_{0}\left(z_{1} e^{i \theta}, \cdots, z_{k} e^{i \theta}\right)=1+e^{i \theta} P_{1}(z)+e^{2 i \theta} P_{2}(z)+\cdots .
$$

Now the volume element $d \omega_{z}$ is invariant under the circular transformation

$$
z_{j} \leftrightarrow z_{j} e^{i \theta} .
$$

Hence the function (5.28) has the same norm as (5.27). Hence both are minimal. But since the development of each starts with 1 and since the minimal functions are unique, (5.27) and (5.28) must be identical. Thus

and

$$
P_{n}(z) \equiv 0, \quad n=1,2,3, \cdots,
$$

$$
f_{0}(z) \equiv 1 .
$$

In a similar manner we see that 


$$
f_{j}(z) \equiv z_{j}, \quad j=1, \cdots, k,
$$

and thus

$$
w_{j}(z) \equiv z_{j}
$$

This yields the desired result.

Now suppose we have a bounded domain $D$ which is equivalent to a bounded proper circular domain $\Delta$. On applying Bergman's theorem, Theorem 8 , we have a procedure for mapping $D$ onto $\Delta$; we merely calculate the $k+1$ minimal functions

$$
f_{\mu}(z), \quad \mu=0,1, \cdots, k,
$$

for $D$ and map by

$$
z_{j}^{\prime}=f_{j}(z) / f_{0}(z), \quad j=1, \cdots, k .
$$

By use of complex orthonormal functions Bergman [6] has simplified the calculation of the minimal functions.

A second important consequence of this result has been derived by Bergman and Welke, namely the corollary of $\$ 2$.

Every origin preserving automorphism of a bounded circular domain $\Gamma$ containing the origin is a homogeneous linear transformation.

Proof. Let

$$
T: \quad z_{j}=a_{1 j} z_{1}^{\prime}+\cdots+a_{k j} z_{k}^{\prime}+\cdots
$$

be an automorphism of $\Delta$. It can be normalized by a homogeneous linear transformation $L$, and this homogeneous linear transformation carries $\Delta$ into a circular domain $\Delta^{\prime}=L \Delta$. Now

$$
\text { TL: } \quad z_{j}=z_{j}^{\prime}+\text { (higher powers) }, \quad j=1, \cdots, k,
$$

is an automorphism of $\Delta^{\prime}$. Since $\Delta^{\prime}$ is circular it is representative and hence the only 1-1 normalized automorphism of $\Delta^{\prime}$ is the identity, that is $T L=I$ or $T=L^{-1}$. Thus $T$ is linear.

6. Mappings of unbounded domains. In this section I want to give briefly and without proof a few results on mappings of unbounded domains. First we shall recall Cartan's theorem, Theorem 1, which states that if we have an inner (analytic) mapping $T$ of a domain $D$ containing the origin onto a bounded domain $D^{\prime}$, and if the linear part of $T$ is the identity, then $T$ is actually the identity.

This theorem does not hold if $D^{\prime}$ is unbounded. In fact, Bieberbach [9], using a result of Fatou $[15,16]$, has shown that there exist two entire functions $z^{\prime}=z+$ (higher powers), $w^{\prime}=w+$ (higher powers), 
which map the entire space $E_{4}$ onto a proper subset of $E_{4}$ in a topological manner. Thus they define an inner mapping whose linear part is the identity and yet the mapping is not the identity.

In spite of this Behnke and Peschl [3] have been able to prove a very beautiful generalization of Cartan's theorem for certain unbounded domains.

Theorem 9 (Behnke ANd Peschl). Let $D$ be a domain containing the origin and let $D$ have the following property: There exist $k$ bounded analytic functions

$$
g_{j}(z)=\sum_{n=n_{j}}^{\infty} P_{n}^{i}(z), \quad j=1, \cdots, k,
$$

in D such that the determinant

$$
J(z)=\partial\left(P_{n_{1}}^{1}, \cdots, P_{n_{k}}^{k}\right) / \partial\left(z_{1}, \cdots, z_{k}\right)
$$

is not identically zero. Then every inner analytic mapping of $D$ whose linear part is the identity is actually the identity.

The development (6.1) is the development of $g_{j}(z)$ in a series of homogeneous polynomials; there is no restriction on the value of the lowest degree $n_{j}$.

Using the result of Behnke and Peschl, Bochner and the speaker [10] have carried over part of the results of $\$ 3$ to a class of unbounded domains. Their result is: If $D$ is not bounded but possesses the alternative property indicated in Theorem 9, if $T$ is an inner mapping of $D$ with the origin as a fixed point, if (3.4) holds, and if the linear parts $\left\{L_{T}, L_{T^{2}}, L_{T^{3}}, \ldots\right\}$ are uniformly bounded in $D$, then $T$ is an automorphism.

7. Concluding remarks. The field of analytic mappings is wide and contains numerous results. It has been impossible to give or even to summarize all of these results; as a consequence many interesting and important results have been omitted. Many of these will be found in the résumé of mapping contained in chapter 7 of the tract by Behnke and Thullen [4]. Several are given in the book by Bergman [6]. I would like to refer the listener to these two books for excellent treatments of various aspects of the theory.

Among the more recent papers on mapping I would like to mention two by Bergman and Spencer $[7,8]$ which have appeared since the publication of the books just mentioned. These papers point in a somewhat different direction from those considered in this talk, and they contain references to other recent treatments. 


\section{BIBLIOGRAPHY}

(The references listed below include only those directly referred to in the talk; much more complete bibliographies are contained in the books by Behnke and Thullen [4] and Bergman [6].)

1. H. Behnke, Über analytische Funktionen mehreren Veränderlichen. II. Natiuliche Grenzen, Abh. Math. Sem. Hamburgischen Univ. vol. 5 (1927) pp. 290-312.

2. - Über analytische Funktionen mehreren Veränderlichen. III. Abbildungen der Kreiskörper, Abh. Math. Sem. Hamburgischen Univ. vol. 7 (1930) pp. 329-341.

3. H. Behnke and E. Peschl, Der Cartansche Eindeutigkeitssatz in unbeschränkten Körpern, Math. Ann. vol. 114 (1937) pp. 69-73.

4. H. Behnke and P. Thullen, Theorie der Funktionen mehreren komplexer Veränderlichen, Ergebnisse der Mathematik und ihrer Grenzgebiete, book 3, number 3, Berlin, 1934.

5. S. Bergman, Über die Existenz von Repräsentantenbereichen, Math. Ann. vol. 102 (1929) pp. 430-446.

6. - Sur les fonctions orthogonales de plusieurs variables complexes avec applications $d$ la theorie des fonctions analytiques, Interscience Publishers, New York, 1941.

7. S. Bergman and D. C. Spencer, On distortion in pseudo-conformal mapping, Trans. Amer. Math. Soc. vol. 51 (1942) pp. 133-163.

8. - $A$ property of pseudo-conformal transformations in the neighborhood of boundary points, Duke Math. J. vol. 9 (1942) pp. 757-762.

9. L. Bieberbach, Beispiel zweier ganzer Funktionen zweier komplexer Variablen, welche eine schlicht volumetreue Abbildung des $R_{4}$ auf einen Teil seiner selbst vermitteln. Preuss. Akad. Wiss. Sitzungsber., 1933.

10. S. Bochner and W. T. Martin, Manuscript of a book on analytic functions of several variables, in preparation.

11. C. Carathéodory, Über die Geometrie der analytischen Abbildungen, Abh. Math. Sem. Hamburgischen Univ. vol. 6 (1928) pp. 96-145.

12. - Über die Abbildungen, die durch Systeme von analytische Funktionen von mehreren Veränderlichen erzeugt werden, Math. Zeit. vol. 34 (1932).

13. H. Cartan, Les fonctions de deux variables complexes et le problème de la reprEsentation analytique, J. Math. Pures Appl. (9) vol. 10 (1931) pp. 1-114.

14. - Sur les fonctions de plus variables complexes. L'itération de transform interieures d'un domaine borne, Math. Zeit. vol. 35 (1932).

15. P. Fatou, Sur les fonctiones méromorphes de deux variables, C. R. Acad. Sci. Paris vol. 175 (1922).

16. - Sur certaines fonctiones uniformes de deux variables, C. R. Acad. Sci. Paris vol. 175 (1922).

17. H. Poincaré, Les fonctions analytiques de deux variables complexes et la representation conforme, Rend. Circ. Mat. Palermo vol. 23 (1907) pp. 185-220.

18. $\mathrm{H}$. Welke, Über die analytischen Abbildungen von Kreiskörpern und Hartogsschen Bereichen, Math. Ann. vol. 103 (1930) pp. 437-449.

19. H. Weyl, Theorie der Darstellung kontinuierlicher halb-einfacher Gruppen durch lineare Transformationen, Math. Zeit. vol. 23 (1925) pp. 271-309.

\section{SyRACUSE UNIVERSITY}

